Full length article

\title{
Effect of short chain fructooligosaccharides (scFOS) on immunological status and gut microbiota of gilthead sea bream (Sparus aurata) reared at two temperatures
}

\author{
Inês Guerreiro a, b, *, Cláudia R. Serra a , Paula Enes ${ }^{\text {a }}$, Ana Couto ${ }^{\text {a }}$, Andreia Salvador ${ }^{\mathrm{c}}$, \\ Benjamín Costas ${ }^{\mathrm{a}}$, Aires Oliva-Teles a, b \\ ${ }^{a}$ CIMAR/CIIMAR - Centro Interdisciplinar de Investigação Marinha e Ambiental, Universidade do Porto, Rua dos Bragas 289, 4050-123 Porto, Portugal \\ b Departamento de Biologia, Faculdade de Ciencias, Universidade do Porto, Rua do Campo Alegre s/n, Ed. FC4, 4169-007 Porto, Portugal \\ ${ }^{\mathrm{c}}$ CEB - Centro de Engenharia Biológica, Universidade do Minho, Campus de Gualtar, 4710-057 Braga, Portugal
}

\section{A R T I C L E I N F O}

\section{Article history:}

Received 17 September 2015

Received in revised form

16 December 2015

Accepted 18 December 2015

Available online 22 December 2015

\section{Keywords:}

Digestive enzymes

Gut histology

Gut microbiota

Immune status

Nitric oxide

Prebiotics

\begin{abstract}
A B S T R A C T
The effects of dietary short chain fructooligosaccharides (scFOS) incorporation on hematology, fish immune status, gut microbiota composition, digestive enzymes activities, and gut morphology, was evaluated in gilthead sea bream (Sparus aurata) juveniles reared at $18{ }^{\circ} \mathrm{C}$ and $25{ }^{\circ} \mathrm{C}$. For that purpose, fish with $32 \mathrm{~g}$ were fed diets including $0,0.1,0.25$ and $0.5 \%$ scFOS during 8 weeks. Overall, scFOS had only minor effects on gilthead sea bream immune status. Lymphocytes decreased in fish fed the $0.1 \%$ scFOS diet. Fish fed the $0.5 \%$ scFOS diet presented increased nitric oxide (NO) production, while total immunoglobulins (Ig) dropped in those fish, but only in the ones reared at $25^{\circ} \mathrm{C}$. Red blood cells, hemoglobin, bactericidal activity and NO were higher at $25{ }^{\circ} \mathrm{C}$, whereas total white blood cells, circulating thrombocytes, monocytes and neutrophils were higher at $18{ }^{\circ} \mathrm{C}$. In fish fed scFOS, lymphocytes were higher at $18^{\circ} \mathrm{C}$. Total Ig were also higher at $18{ }^{\circ} \mathrm{C}$ but only in fish fed $0.1 \%$ and $0.5 \%$ scFOS diets. No differences in gut bacterial profiles were detected by PCR-DGGE (polymerase chain reaction denaturing gradient gel electrophoresis) between dietary treatments. However, group's similarity was higher at $25^{\circ} \mathrm{C}$. Digestive enzymes activities were higher at $25^{\circ} \mathrm{C}$ but were unaffected by prebiotics incorporation. Gut morphology was also unaffected by dietary prebiotic incorporation.

Overall, gut microbiota composition, digestive enzymes activities and immunity parameters were affected by rearing temperature whereas dietary scFOS incorporation had only minor effects on these parameters. In conclusion, at the tested levels scFOS does not seem worthy of including it in gilthead sea bream juveniles diets.
\end{abstract}

() 2015 Elsevier Ltd. All rights reserved.

\section{Introduction}

Prebiotics can be defined as non-digestible fibers that potentially increase specific-health promoting gut bacteria in the host [1]. Thus, prebiotics can positively affect host's health either indirectly, through by-products produced during bacterial prebiotic fermentation, or directly, through prebiotics interaction with pattern recognition receptors [2].

Fructooligosaccharides (FOS) are one of the most studied prebiotics in humans, farm animals and fish [2-5]. Short-chain

\footnotetext{
* Corresponding author. Departamento de Biologia, Faculdade de Ciências, Universidade do Porto, Rua do Campo Alegre s/n, Ed. FC4, 4169-007 Porto, Portugal

E-mail address: imsguerreiro@gmail.com (I. Guerreiro).
}

fructooligosaccharides (scFOS) are similar to FOS but with a lower degree of polymerization, ranging from 1 to 5 fructose oligomers [6]. ScFOS are however much less studied than FOS. In fish, scFOS has been only evaluated in turbot (Scophthalmus maximus), European sea bass (Dicentrarchus labrax), gilthead sea bream (Sparus aurata), common carp (Cyprinus carpio), and hybrid tilapia (Oreochromis niloticus $+\times$ Oreochromis aureus ơ) [7-14].

FOS is known to support growth and survival of gastrointestinal tract autochthonous bacteria, such as members of the genus Lactobacillus, which possess $\beta$-fructosidase activity and thus can hydrolyse FOS $\beta$-(2-1) glycosidic bonds [3]. Lactobacillus is known to interact with the host immune system, but the precise mechanisms involved are not completely clarified. Nonetheless, it seems that Lactobacillus, or its end-metabolic products, interact with gut 
epithelial cells, macrophages, dendritic cells, and lymphocytes [15]. For instance, in mice given Lactobacillus as a probiotic, the gut mucosal immune system was affected mainly through activation of innate immune response cells [16]. FOS is also considered an immunosaccharide [2] as it has a direct signaling capacity on human's immune cells, by activating toll-like receptors, mainly TLR2 and, to a lesser extent, TLR4 [17]. Although the benefits of prebiotics to animals are well known, as well as the relationship between gut bacteria and host's immune system, the majority of studies on prebiotics effects in fish immune status does not simultaneously evaluate gut microbial composition [18-23]. It was, however, recently reported an increase in gut cultivable lactic acid bacteria (LAB) population and a stimulation of several immune parameters upon incorporation of FOS in the diets for stellate sturgeon (Acipenser stellatus) and common carp (C. carpio) [24,25].

Prebiotics effects on fish immunity, particularly of FOS, are reported as immunomodulatory [2-5]. For instance, blunt snout bream (Megalobrama amblycephala) fed a diet with $0.4 \%$ FOS presented lower levels of plasma cortisol and higher levels of immunoglobulin, lysozyme, plasma acid phosphatase, alternative complement activity and nitrogen monoxide than fish fed the control diet [23]. Triangular bream (Megalobrama terminalis) fed a diet with $0.6 \%$ FOS had increased leucocyte counts, plasma alternative complement activity and immunoglobulins compared to fish fed the control diet [22]. Red drum (Sciaenops ocellatus) fed a diet with $1 \%$ FOS had increased plasma lysozyme [26]. In Pacific white shrimp (Litopenaeus vannamei), diets including scFOS led to alterations in gut microbiota and enhanced total hemocyte count and hemocyte respiratory burst [27].

In gilthead sea bream, prebiotics effects on immune parameters were so far only evaluated for inulin and mannanoligosaccharides (MOS). MOS did not affect fish health indicators, whereas leucocytes phagocytic capacity was decreased in fish fed inulin for 1 week [18,21]. In another study, complement activity, leukocyte phagocytic ability and capacity increased after 2 weeks of feeding an inulin supplemented diet [28]. However, after 4 weeks of feeding the same diet, differences in leucocytes phagocytic ability and capacity disappeared while gut bacteria richness was reduced $[28,29]$.

Dietary supplementation with FOS has been associated with an increase in digestive enzymes activity in some fish species, which may be correlated with alterations in gut microbiota [30-32]. Although, the effect of FOS on gilthead sea bream digestive enzymes was not yet evaluated, MOS was associated with increased protein, carbohydrates and energy digestibility's [33].

Prebiotic effects on fish gut morphology are extensively studied. MOS was reported to increase gut absorptive area through increased microvilli length and density [34-38]. FOS was also reported to induce changes in morphology of fish intestine, such as increased microvillus height [26,32]. However, inulin was reported to induce significant damage in gilthead sea bream gut [29].

Fish, as heterothermic animals, are heavily influenced by environmental conditions and, as suggested by Ringø et al. [3], temperature may have greater effects than diet in fish health. This may turn difficult an evaluation of prebiotics effect. Indeed, water temperature was already reported to influence immunological parameters and gut bacterial community [9,12,39-42].

Several studies on the effects of prebiotics $[2,9,43]$ and rearing temperature $[9,41,42]$ on fish immune status are already available. However, studies simultaneously evaluating both parameters and their potential interactive effects on fish immune status are very scarce [9].

Since prebiotics are reported to promote gastrointestinal health and immunological status [44], the study of prebiotic effects on gut function and integrity, and on immunological parameters are particularly important. Therefore, the aim of this study was to evaluate the effect of dietary scFOS supplementation in the hematological profile, fish immune status, allochthonous gut microbiota composition, digestive enzymes activities, and gut morphology of gilthead sea bream juveniles reared at two temperatures: 18 and $25^{\circ} \mathrm{C}$.

\section{Material and methods}

\subsection{Diets composition}

Four diets were formulated to be isolipidic (18\% lipid) and isonitrogenous (46\% protein). Fish meal and plant feedstuffs (soybean meal, wheat gluten, corn gluten and wheat meal) were used as protein sources (circa 50\% protein from fish meal and 50\% from plant feedstuffs), and fish oil was the main lipid source. The experimental diets included $0 \%$ (diet D0 - control diet), $0.1 \%$ (diet D0.1), 0.25\% (diet D0.25), and 0.5\% (diet D0.5) of scFOS (PROFEED Maxflow, Jefo, France) replacing $\alpha$-cellulose. All ingredients were thoroughly mixed and dry pelleted in a laboratory pellet mill (California Pellet Mill, CPM Crawfordsville, IN, USA), through a $2.0 \mathrm{~mm}$ die. Pellets were dried in an oven at $40^{\circ} \mathrm{C}$ for $48 \mathrm{~h}$, and then stored in a freezer in airtight bags until use. Ingredients and proximate composition of the experimental diets are presented in Table 1.

Chemical analyses of the diets were performed following the Association of Official Analytical Chemists methods [45]. Dietary starch content was determined according to Beutler [46].

\subsection{Growth trial}

The experiment was performed at the Marine Zoology Station, Porto University, Portugal, with gilthead sea bream (S. aurata) juveniles obtained from a commercial fish farm (Maresa S.A., Ayamonte, Huelva, Spain). The trial was performed in 2 identical recirculating water systems, each equipped with 12 cylindrical fiberglass tanks of $100 \mathrm{~L}$ water capacity, and thermo-regulated to $18.0 \pm 0.5^{\circ} \mathrm{C}$ and $25.0 \pm 0.6{ }^{\circ} \mathrm{C}$, respectively. The tanks were supplied with a continuous flow of filtered seawater $\left(2.5-3.5 \mathrm{~L} \mathrm{~min}^{-1}\right)$ of $35 \pm 1 \mathrm{~g} \mathrm{~L}^{-1}$ salinity, and dissolved oxygen was kept near saturation $\left(7 \mathrm{mg} \mathrm{L}^{-1}\right)$. After a quarantine period of $1 \mathrm{month}$, fish were transferred to the experimental systems and adapted to the experimental conditions for 15 days. During quarantine and adaptation periods, fish were fed a commercial diet (48\% protein and 17\% lipids; Sorgal, S.A. Ovar, Portugal). A total of 528 fish with an initial mean body weight of $32.0 \pm 0.01 \mathrm{~g}$ were randomly distributed by the tanks, 22 fish per tank. The experimental diets were randomly assigned to triplicate groups within each temperature, hence 6 tanks per dietary treatment, with 3 tanks at each temperature. The trial lasted 8 weeks, and during that period fish were fed by hand, twice daily, 6 days a week, until apparent visual satiation. Utmost care was taken to avoid feed losses. The experiment was performed by accredited scientists (following FELASA category $C$ recommendations) and was conducted according to the European Union directive 2010/63/EU on the protection of animals for scientific purposes.

\subsection{Sampling}

At the end of the trial, a total of 8 fish per tank were randomly sampled $4 \mathrm{~h}$ after the morning meal. Blood from 3 fish was collected from the caudal vein using heparinized syringes and placed in heparinized tubes. One aliquot was used for hematological assessment while the remaining blood was centrifuged at $3000 \times \mathrm{g}$ for $10 \mathrm{~min}$ at room temperature. The resulting plasma was 
Table 1

Ingredients and proximate composition of the experimental diets.

\begin{tabular}{|c|c|c|c|c|}
\hline & \multicolumn{4}{|l|}{ Diets } \\
\hline & D0 & D0.1 & D0.25 & D0.5 \\
\hline \multicolumn{5}{|c|}{ Ingredients (\% dry weight) } \\
\hline Fish meal $^{\mathrm{a}}$ & 31.4 & 31.4 & 31.4 & 31.4 \\
\hline Corn gluten $^{\mathrm{b}}$ & 5.0 & 5.0 & 5.0 & 5.0 \\
\hline Wheat gluten ${ }^{c}$ & 5.0 & 5.0 & 5.0 & 5.0 \\
\hline Wheat meal ${ }^{\mathrm{d}}$ & 15.2 & 15.2 & 15.2 & 15.2 \\
\hline Soy meal ${ }^{\mathrm{e}}$ & 25.0 & 25.0 & 25.0 & 25.0 \\
\hline $\operatorname{scFOS}^{\mathrm{f}}$ & - & 0.1 & 0.25 & 0.5 \\
\hline Cellulose $^{g}$ & 0.5 & 0.4 & 0.25 & - \\
\hline Cod liver oil & 13.7 & 13.7 & 13.7 & 13.7 \\
\hline Bicalcium phosphate $^{\mathrm{h}}$ & 0.7 & 0.7 & 0.7 & 0.7 \\
\hline Vitamin mix ${ }^{i}$ & 1.0 & 1.0 & 1.0 & 1.0 \\
\hline Mineral mix ${ }^{j}$ & 1.0 & 1.0 & 1.0 & 1.0 \\
\hline Choline chloride (50\%) & 0.5 & 0.5 & 0.5 & 0.5 \\
\hline Binder (Aquacube) ${ }^{\mathrm{k}}$ & 1.0 & 1.0 & 1.0 & 1.0 \\
\hline \multicolumn{5}{|c|}{ Proximate analysis (\% dry weight) } \\
\hline Dry matter & 87.4 & 87.3 & 89.1 & 88.5 \\
\hline Crude protein & 45.8 & 46.6 & 45.7 & 46.6 \\
\hline Crude lipids & 18.7 & 18.0 & 18.0 & 18.3 \\
\hline Ash & 9.3 & 9.1 & 9.2 & 9.3 \\
\hline Starch & 10.8 & 10.5 & 11.4 & 11.1 \\
\hline Gross energy $\left(\mathrm{kJ} \mathrm{g}^{-1}\right)^{1}$ & 20.1 & 19.9 & 19.9 & 20.1 \\
\hline
\end{tabular}

DM, dry matter; $C P$, crude protein; $C L$, crude lipid.

a Steam Dried LT fish meal, Pesquera Diamante, Austral Group, S.A Perú (CP: 71.7\% DM; CL: 9.5\% DM).

b Sorgal, S.A. Ovar, Portugal (CP: 72.2\% DM; CL: 2.0\% DM).

c Sorgal, S.A. Ovar, Portugal (CP: 84.4\% DM; CL: 1.8\% DM).

d Sorgal, S.A. Ovar, Portugal (CP: 14.1\% DM; CL: 3.2\% DM).

e Sorgal, S.A. Ovar, Portugal (CP: 50.2\% DM; CL: 2.4\% DM).

f PROFEED Maxflow "Fructo-Oligosaccharides" (Jefo, France).

g Sigma-Aldrich, Sintra, Portugal.

h Premix, Portugal (Calcium: 24\%; Total phosphorus: 18\%).

${ }^{\mathrm{i}}$ Vitamins ( $\mathrm{mg} \mathrm{kg}^{-1}$ diet): retinol acetate, 18000 (IU kg-1 diet); cholecalciferol, 2000 (IU kg-1 diet); alpha tocopherol acetate, 35; sodium menadione bisulphate, 10; thiamin- $\mathrm{HCl}, 15$; riboflavin, 25; calcium pantothenate, 50; nicotinic acid, 200; pyridoxine $\mathrm{HCl}$, 5; folic acid 10; cyanocobalamin, 0.02 ; biotin, 1.5; ascorbic acid, 50; inositol, 400.

j Minerals ( $\mathrm{mg} \mathrm{kg}^{-1}$ diet): cobalt sulphate, 1.91; copper sulphate, 19.6; iron sulphate, 200; sodium fluoride, 2.21; potassium iodide, 0.78; magnesium oxide, 830; manganese oxide, 26; sodium selenite, 0.66; zinc oxide, 37.5; dibasic calcium phosphate, 5.93 ( $\mathrm{g} \mathrm{kg}^{-1}$ diet); potassium chloride, 1.15 ( $\mathrm{g} \mathrm{kg}^{-1}$ diet); sodium chloride, $0.40\left(\mathrm{~g} \mathrm{~kg}^{-1}\right.$ diet $)$.

k Agil, England (guar gum, polymethyl carbamide, manioc starch blend, hydrate calcium sulphate).

${ }^{1}$ Gross energy calculated based on theoretical values (CP: $23.6 \mathrm{~kJ} \mathrm{~g}^{-1}$; CL: $39.5 \mathrm{~kJ} \mathrm{~g}^{-1}$; Carbohydrates: $17.2 \mathrm{~kJ} \mathrm{~g}^{-1}$.

frozen at $-80{ }^{\circ} \mathrm{C}$ for immune parameters analyses. After blood collection fish were euthanized with a sharp blow in the head. Gut with intestinal content was removed and divided in 2 parts, anterior and posterior gut, immediately frozen in liquid nitrogen and then stored at $-80{ }^{\circ} \mathrm{C}$ until measurement of digestive enzymes activities. The anterior gut was defined as the region from the last pyloric caeca and gut mid length. The posterior gut was defined as the second half of the gut.

Three other fish were euthanized with a sharp blow in the head, and gut was dissected on chilled trays and freed from the adjacent adipose and connective tissues. Circa $1 \mathrm{~cm}$ of the middle regions of the anterior and posterior gut were collected for histological evaluation. The samples were rinsed in phosphate buffered saline (PBS), carefully blotted dry with a paper towel, immediately fixed in phosphate buffered formalin (4\%, pH 7.4) for $24 \mathrm{~h}$ and then transferred to ethanol (70\%) until further processing.

Two other fish per tank were sampled under aseptic conditions for digesta collection. Digesta was collected by squeezing the entire gut, immediately frozen in liquid nitrogen and then stored at $-80{ }^{\circ} \mathrm{C}$ until characterization of allochthonous microbiota.

\subsection{Hematological analysis}

Fresh heparinized blood was used for hematocrit (Ht) and hemoglobin ( $\mathrm{Hb}$ ) determination, and for blood cells counts. $\mathrm{Ht}, \mathrm{Hb}$, total red blood cells (RBC), white blood cells (WBC) and differential white blood cell counts were determined as described by Guerreiro et al. [9]. The mean corpuscular volume (MCV), mean corpuscular hemoglobin $(\mathrm{MCH})$ and mean corpuscular hemoglobin concentration $(\mathrm{MCHC})$ were calculated as follows:

$$
\begin{aligned}
& \operatorname{MCV}\left(\mu \mathrm{m}^{3}\right)=(\mathrm{Ht} / \mathrm{RBC}) \times 10 \\
& \operatorname{MCH}\left(\operatorname{pg~cell}{ }^{-1}\right)=(\mathrm{Hb} / \mathrm{RBC}) \times 10 \\
& \operatorname{MCHC}\left(\mathrm{g} 100 \mathrm{ml}^{-1}\right)=(\mathrm{Hb} / \mathrm{Ht}) \times 100
\end{aligned}
$$

\subsection{Immune parameters}

Bactericidal and anti-protease activities, nitric oxide (NO), and total immunoglobulins (Ig) in plasma were determined as described by Guerreiro et al. [9] and Machado et al. [47].

\subsection{Microbial diversity}

Samples of 2 fish per tank were pooled to reduce variation. DNA was extracted from $300 \mathrm{mg}$ of gut contents as described in Pitcher et al. [48]. Bacterial 16S rRNA gene fragments were amplified by a touchdown PCR on a T100 ${ }^{\mathrm{TM}}$ Thermal Cycler (Bio-Rad Laboratories Lda., Amadora, Portugal), using oligonucleotide primers $16 \mathrm{~S}-358 \mathrm{~F}$ (which contained a GC clamp at the 5énd) and 16S-517R [49]. $300 \mathrm{ng}$ of each PCR product were resolved on $8 \%$ polyacrylamide gel composed by a denaturing gradient of $40-80 \% 7 \mathrm{M}$ urea $/ 40 \%$ formamide. Electrophoresis was run on a DCode ${ }^{\mathrm{TM}}$ universal mutation detection system (Bio-Rad Laboratories Lda., Amadora, Portugal) during $16 \mathrm{~h}$ at $60{ }^{\circ} \mathrm{C}, 65 \mathrm{~V}$ in $1 \times$ TAE buffer. Gels were stained for $1 \mathrm{~h}$ with SYBR-Gold Nucleic Acid Gel Stain (Thermo Fisher Scientific Inc.) and imaged on a Gel Doc EZ System (Bio-Rad Laboratories Lda., Amadora, Portugal). Distinct bands were excised from the gel and eluted in $20 \mu \mathrm{l}$ ultrapure water prior to DNA reamplification [49] using the same oligonucleotide primers as above, but without the GC clamp. Amplicons were sequenced to identify microbiota OTUs (Operational Taxonomic Units). Phylogenetic analysis, to identify the closest known species, was done by comparison with sequences in the GenBank non-redundant nucleotide database using BLAST (http://www.ncbi.nlm.nih.gov) (Macrogen Europe, Amsterdam, The Netherlands). Only sequences higher than 100 bp reads and 80-100\% query coverage were considered a valid identification.

\subsection{Digestive enzymes activities}

Each gut section was homogenized in 10 parts of ice-cold $50 \mathrm{mM}$ Tris- $\mathrm{HCl}$ buffer $\mathrm{pH}$ 7.5. Homogenates were centrifuged at $33000 \times \mathrm{g}$ for $15 \mathrm{~min}$ at $4{ }^{\circ} \mathrm{C}$ and the resultant supernatants were used for digestive enzyme assays.

Total alkaline protease (TAP), lipase (EC3.1.1.3) and $\alpha$-amylase (EC3.2.1.1) activities, and protein concentrations were measured as described in Guerreiro et al. [12].

All enzyme activities were expressed as specific activity $\left(\mathrm{U} \mathrm{mg}^{-1}\right.$ of soluble protein for TAP and $\mathrm{mU} \mathrm{mg}^{-1}$ for lipase and $\alpha$-amylase).

\subsection{Histological processing and morphological evaluation}

Gut samples were processed and evaluated as described in Couto et al. [50] by visualization with a Carl Zeiss MicroImaging GmbH model Primo Star (Jena, Germany). Briefly, blinded 
evaluation was performed using a semi-quantitative scoring system ranging from 1 to 5 (Table 2). Score 1 was given to the normal tissue appearance and subsequent scores accounted for increasing alterations in tissue histomorphology. Gut samples were evaluated according to the criteria suggested by Krogdahl et al. [51]: widening and shortening of the gut folds, loss of supranuclear vacuolization in absorptive cells (enterocytes) of the gut epithelium, nucleus position in the enterocyte, widening of lamina propria within gut folds, infiltration of mixed leucocyte population in lamina propria and submucosa. The overall score of histomorphological alterations was calculated by averaging scores of the separate parameters analyzed.

\subsection{Statistical analysis}

Data are presented as means \pm standard deviation. Statistical analysis of hematological and immune parameters, and of digestive enzymes was done by two-way ANOVA, with diet and temperature as factors, using a SPSS 21 software package for Windows (IBM ${ }^{\circledR}$ SPSS ${ }^{\circledR}$ Statistics, New York, USA). Data were tested for normality by the Shapiro-Wilk test and for homogeneity of variances by the Levene's test. When normality was not verified data were transformed prior to ANOVA. In case of interaction between factors $(\mathrm{Ht}$, lymphocytes, Ig, and posterior gut TAP), one-way ANOVA was performed for prebiotic level within each temperature. Significant differences among dietary groups were determined by the Tukey's multiple range test. In the case of interaction, t-tests were performed to assess differences between temperatures within the same diet. Histological data was neither normal nor homogeneous and could not be normalized, thus Kruskal-Wallis non-parametric tests and subsequent pairwise comparison were performed. The probability level of 0.05 was used for rejection of the null hypothesis in all tests.

DGGE (Denaturing Gradient Gel Electrophoresis) banding patterns were transformed into presence/absence matrices and band intensities were measured using Quantity One 1-D Analysis Software v4.6.9 (Bio-Rad Laboratories Lda., Amadora, Portugal). Relative similarities between dietary treatments and replicates were calculated using Primer software v7.0.5 (PRIMER-E Ltd, Ivybridge, UK). Similarity percentages (SIMPER) were used to represent the relative similarities between treatments. Species richness was assessed using Margalef's measure of richness, and species diversity was assessed by the Shannon-Weaver index. Clustering of DGGE patterns was achieved by construction of dendrograms using the unweighted pair group method with arithmetic averages (UPGMA). Microbial diversity parameters were subjected to twoway ANOVA, with temperature and diet as fixed factors.

\section{Results}

\subsection{Hematological parameters}

Dietary prebiotic incorporation did not affect total blood cell counts, $\mathrm{Ht}, \mathrm{Hb}, \mathrm{MCV}, \mathrm{MCH}$, and $\mathrm{MCHC}$ (Table 3). $\mathrm{MCV}, \mathrm{MCH}$, and $\mathrm{MCHC}$ were also unaffected by rearing temperature. In contrast, $\mathrm{RBC}$, and $\mathrm{Hb}$ were higher in fish reared at $25{ }^{\circ} \mathrm{C}$ whereas the opposite was observed for WBC. Ht was also higher in fish reared at $25^{\circ} \mathrm{C}$ for all diets ( $\mathrm{t}$-test $\mathrm{P}<0.05$ ), except diet D0.1 (t-test $\mathrm{P}>0.05$ ).

Differential WBC counts are presented in Table 4 . Thrombocytes, monocytes and neutrophils were higher in fish reared at $18^{\circ} \mathrm{C}$ than at $25^{\circ} \mathrm{C}$. In fish fed scFOS diets ( $\mathrm{t}$-test $\mathrm{P}<0.05$ ), but not the control diet (t-test $\mathrm{P}>0.05$ ), lymphocytes were higher at $18{ }^{\circ} \mathrm{C}$ than at

Table 2

Continuous scale scoring system with the range of tissue scores set at $1-5,5$ indicating major alterations [52].

\begin{tabular}{|c|c|c|}
\hline & \multicolumn{2}{|l|}{ Score range } \\
\hline & 1 & to 5 \\
\hline Gut folds & Tall and distinct & Short, indistinct, fused \\
\hline Lamina propria width and cellularity & Thin, low cellularity & Markedly widened and increased cellularity \\
\hline Submucosa width and cellularity & Thin, low cellularity & Markedly widened and increased cellularity \\
\hline Intraepithelial leucocytes infiltration & Low infiltration & Highly infiltrated \\
\hline Enterocytes vacuolization & Well defined, regular vacuoles size & Absent or hypervacuolated; irregular vacuoles size \\
\hline Enterocytes nucleus position & Basal & Apical \\
\hline
\end{tabular}

Table 3

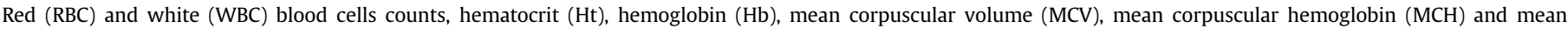
corpuscular hemoglobin concentration $(\mathrm{MCHC})$ of gilthead sea bream fed the experimental diets at two temperatures.

\begin{tabular}{|c|c|c|c|c|c|c|c|c|}
\hline & \multirow[t]{2}{*}{ Temperature } & \multicolumn{4}{|l|}{ Diets } & \multicolumn{3}{|c|}{ Two-way ANOVA } \\
\hline & & D0 & D0.1 & D0.25 & D0.5 & Temperature & Diets & Interaction \\
\hline \multirow[t]{2}{*}{$\operatorname{RBC}\left(\times 10^{6} \mathrm{~mm}^{-3}\right)$} & $18^{\circ} \mathrm{C}$ & $2.17 \pm 0.27$ & $2.88 \pm 1.21$ & $2.21 \pm 0.38$ & $2.43 \pm 0.22$ & $*$ & ns & ns \\
\hline & $25^{\circ} \mathrm{C}$ & $2.68 \pm 0.28$ & $2.65 \pm 0.31$ & $2.50 \pm 0.53$ & $2.76 \pm 0.62$ & & & \\
\hline \multirow[t]{2}{*}{ Ht (\%) } & $18^{\circ} \mathrm{C}$ & $23.7 \pm 2.1$ & $26.3 \pm 3.2$ & $25.4 \pm 2.3$ & $26.6 \pm 1.8$ & $* * *$ & ns & $*$ \\
\hline & $25^{\circ} \mathrm{C}$ & $29.1 \pm 2.0$ & $27.2 \pm 3.1$ & $30.4 \pm 1.4$ & $29.6 \pm 2.9$ & & & \\
\hline \multirow[t]{2}{*}{$\mathrm{Hb}\left(\mathrm{g} \mathrm{dl}^{-1}\right)$} & $18^{\circ} \mathrm{C}$ & $6.06 \pm 0.47$ & $6.36 \pm 0.67$ & $6.43 \pm 0.59$ & $6.50 \pm 0.44$ & $* * *$ & ns & ns \\
\hline & $25^{\circ} \mathrm{C}$ & $7.19 \pm 0.65$ & $7.00 \pm 0.91$ & $7.07 \pm 1.12$ & $7.23 \pm 1.44$ & & & \\
\hline \multirow[t]{2}{*}{$\operatorname{MCV}\left(\mu \mathrm{m}^{3}\right)$} & $18^{\circ} \mathrm{C}$ & $110.1 \pm 13.1$ & $99.5 \pm 31.7$ & $115.4 \pm 16.5$ & $110.1 \pm 11.1$ & ns & ns & ns \\
\hline & $25^{\circ} \mathrm{C}$ & $109.4 \pm 10.5$ & $103.8 \pm 13.2$ & $129.7 \pm 42.7$ & $109.5 \pm 16.1$ & & & \\
\hline \multirow[t]{2}{*}{$\mathrm{MCH}\left(\mathrm{pg}\right.$ cell $\left.^{-1}\right)$} & $18^{\circ} \mathrm{C}$ & $28.2 \pm 3.0$ & $24.0 \pm 7.4$ & $29.3 \pm 5.0$ & $26.9 \pm 2.9$ & ns & ns & ns \\
\hline & $25^{\circ} \mathrm{C}$ & $27.0 \pm 2.6$ & $26.6 \pm 3.3$ & $28.8 \pm 3.5$ & $26.4 \pm 3.9$ & & & \\
\hline \multirow[t]{2}{*}{$\mathrm{MCHC}\left(\mathrm{g} 100 \mathrm{~mL}^{-1}\right)$} & $18^{\circ} \mathrm{C}$ & $25.7 \pm 1.1$ & $24.3 \pm 0.8$ & $25.4 \pm 1.6$ & $24.5 \pm 1.4$ & ns & ns & ns \\
\hline & $25^{\circ} \mathrm{C}$ & $24.7 \pm 1.3$ & $25.7 \pm 2.0$ & $23.2 \pm 3.4$ & $24.3 \pm 3.5$ & & & \\
\hline \multirow[t]{2}{*}{$\mathrm{WBC}\left(\times 10^{4} \mathrm{~mm}^{-3}\right)$} & $18^{\circ} \mathrm{C}$ & $14.7 \pm 5.0$ & $15.4 \pm 3.4$ & $15.8 \pm 2.8$ & $14.9 \pm 2.3$ & $* * *$ & ns & ns \\
\hline & $25^{\circ} \mathrm{C}$ & $6.01 \pm 0.63$ & $5.92 \pm 0.58$ & $6.46 \pm 1.48$ & $6.02 \pm 1.16$ & & & \\
\hline
\end{tabular}

Values presented as means \pm standard deviation $(n=9)$.

${ }^{*} \mathrm{P}<0.05 ;{ }^{* * *} \mathrm{P}<0.001$; ns: not significant.

a Two-way ANOVA: if interaction was significant, one-way ANOVA was performed for diets within each temperature. 
Table 4

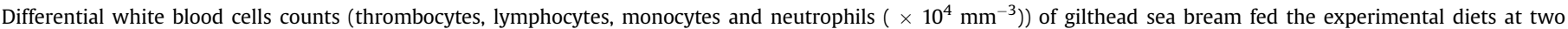
temperatures.

\begin{tabular}{|c|c|c|c|c|c|c|c|c|}
\hline & \multirow[t]{2}{*}{ Temperature } & \multicolumn{4}{|l|}{ Diets } & \multicolumn{3}{|c|}{ Two-way ANOVA } \\
\hline & & D0 & D0.1 & D0.25 & D0.5 & Temperature & Diets & Interaction \\
\hline \multirow[t]{2}{*}{ Thrombocytes } & $18^{\circ} \mathrm{C}$ & $11.1 \pm 4.3$ & $11.7 \pm 3.0$ & $12.2 \pm 3.0$ & $11.5 \pm 1.8$ & \multirow[t]{2}{*}{$* * *$} & \multirow[t]{2}{*}{ ns } & \multirow[t]{2}{*}{ ns } \\
\hline & $25^{\circ} \mathrm{C}$ & $3.43 \pm 1.11$ & $4.30 \pm 0.82$ & $4.50 \pm 1.46$ & $4.13 \pm 0.60$ & & & \\
\hline \multirow[t]{2}{*}{ Lymphocytes } & $18^{\circ} \mathrm{C}$ & $1.77 \pm 0.78$ & $2.37 \pm 0.89$ & $2.11 \pm 0.96$ & $2.22 \pm 0.76$ & \multirow[t]{2}{*}{$* * *$} & \multirow[t]{2}{*}{ ns } & \multirow[t]{2}{*}{$* *$} \\
\hline & $25^{\circ} \mathrm{C}$ & $1.89 \pm 0.66 b$ & $0.96 \pm 0.54 \mathrm{a}$ & $1.19 \pm 0.51 \mathrm{ab}$ & $1.26 \pm 0.81 \mathrm{ab}$ & & & \\
\hline \multirow[t]{2}{*}{ Monocytes } & $18^{\circ} \mathrm{C}$ & $0.81 \pm 0.89$ & $0.47 \pm 0.29$ & $0.62 \pm 0.41$ & $0.44 \pm 0.20$ & \multirow[t]{2}{*}{$* * *$} & \multirow[t]{2}{*}{ ns } & \multirow[t]{2}{*}{$\mathrm{ns}$} \\
\hline & $25^{\circ} \mathrm{C}$ & $0.23 \pm 0.07$ & $0.21 \pm 0.11$ & $0.21 \pm 0.13$ & $0.16 \pm 0.10$ & & & \\
\hline \multirow[t]{2}{*}{ Neutrophils } & $18^{\circ} \mathrm{C}$ & $1.00 \pm 0.73$ & $0.91 \pm 0.40$ & $0.84 \pm 0.48$ & $0.72 \pm 0.53$ & \multirow[t]{2}{*}{$* * *$} & \multirow[t]{2}{*}{ ns } & \multirow[t]{2}{*}{ ns } \\
\hline & $25^{\circ} \mathrm{C}$ & $0.45 \pm 0.09$ & $0.44 \pm 0.47$ & $0.56 \pm 0.22$ & $0.47 \pm 0.32$ & & & \\
\hline
\end{tabular}

Values presented as means \pm standard deviation $(\mathrm{n}=9) . '$

${ }^{* *} \mathrm{P}<0.01 ;{ }^{* * *} \mathrm{P}<0.001$; ns: not significant.

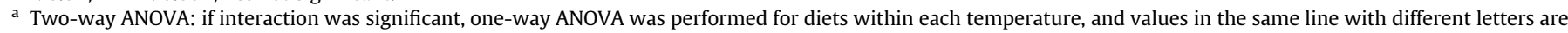
significantly different $(\mathrm{P}<0.05)$

$25^{\circ} \mathrm{C}$. Further, at $25^{\circ} \mathrm{C}$, lymphocytes decreased in fish fed diet D0.1 compared to fish fed the control diet.

\subsection{Immune parameters}

Plasma anti-protease activity was unaffected by rearing temperature or prebiotic supplementation, while plasma bactericidal activity and $\mathrm{NO}$ were higher in fish reared at $25^{\circ} \mathrm{C}$ (Table 5). Plasma total Ig was also affected by rearing temperature, however due to interaction between factors, temperature affected diets differently. Thus, Ig was higher at $18{ }^{\circ} \mathrm{C}$ than at $25^{\circ} \mathrm{C}$ in fish fed diets D0.1 and D0.5 (t-test $\mathrm{P}<0.05$ ), whereas temperature had no effect in fish fed the control and D0.25 diets (t-test $\mathrm{P}>0.05$ ). NO production was higher in fish fed diet D0.5 than the control and D0.25 diets. At $18{ }^{\circ} \mathrm{C}$ plasma total Ig was not affect by diet composition, while at $25^{\circ} \mathrm{C}$ Ig was lower in fish fed diet D0.5 than the control and D0.25 diets.

\subsection{Microbial diversity}

The Bray-Curtis dendrogram and V3 16S rRNA DGGE fingerprints of bacterial communities indicate that the allochthonous (Fig. 1) population had more similar profiles at $25{ }^{\circ} \mathrm{C}$ (similarity percentages $>60 \%$ ) than at $18{ }^{\circ} \mathrm{C}$ ( similarity percentages $\left.\approx 50 \%\right)$. Moreover, at $25{ }^{\circ} \mathrm{C}$ dietary treatments appeared to be clustered, while at $18{ }^{\circ} \mathrm{C}$ dietary treatments clusters were not observed.

The average number of OTUs, microbial richness, diversity, and similarity were not affected by dietary prebiotic (Table 6). Similarity was the only parameter affected by rearing temperature, with higher similarity registered in fish reared at $25^{\circ} \mathrm{C}$.
Sequence analysis from the DGGE bands (Table 7) showed that the detected dominant allochthonous bacteria were closely related to uncultured bacteria previously isolated from weaned piglets, wild and domesticated adult black tiger shrimp (Penaeus monodon), hybrid tilapia (Tilapia nilotica $\times T$. aurea), and Canada goose feces. Three OTUs were most closely related to Lactobacillus sp., Oceanobacillus sp. and Cyanothece sp., while thirteen OTUs were not successfully sequenced.

\subsection{Digestive enzymes}

TAP, $\alpha$-amylase and lipase activities in anterior and posterior gut are presented in Table $8 \alpha$-amylase and lipase activities were higher in fish reared at $25^{\circ} \mathrm{C}$, in both anterior and posterior gut. TAP activity was not affected by temperature in the anterior gut, but it was higher at $25^{\circ} \mathrm{C}(\mathrm{t}$-test $\mathrm{P}<0.05)$ in the posterior gut, except for fish fed diet D0.1 ( $t$-test $\mathrm{P}>0.05$ ). TAP in the posterior gut was the only digestive enzyme affected by prebiotic incorporation. Thus, at $18^{\circ} \mathrm{C}$ TAP activity was higher in fish fed diet D0.1 compared to fish fed diet D0.5.

\subsection{Gut morphology}

The average scores of parameters used to assess gut morphology were unaffected by prebiotic incorporation or rearing temperature (Table 9).

\section{Discussion}

It is known that interactions between gut bacteria and host

Table 5

Plasma immune humoral parameters of gilthead sea bream fed the experimental diets at two temperatures.

\begin{tabular}{|c|c|c|c|c|c|c|c|c|}
\hline & \multirow[t]{2}{*}{ Temperature } & \multicolumn{4}{|l|}{ Diets } & \multicolumn{3}{|c|}{ Two-way ANOVA ${ }^{a}$} \\
\hline & & D0 & D0.1 & D0.25 & D0.5 & Temperature & Diets & Interaction \\
\hline \multirow[t]{2}{*}{ Bactericidal Activity (\%) } & $18^{\circ} \mathrm{C}$ & $45.7 \pm 8.0$ & $46.6 \pm 2.9$ & $44.6 \pm 6.0$ & $42.5 \pm 5.3$ & \multirow[t]{2}{*}{$* * *$} & \multirow[t]{2}{*}{ ns } & \multirow[t]{2}{*}{ ns } \\
\hline & $25^{\circ} \mathrm{C}$ & $59.6 \pm 8.9$ & $57.2 \pm 7.3$ & $63.9 \pm 8.2$ & $59.4 \pm 8.1$ & & & \\
\hline \multirow[t]{2}{*}{ Nitric oxide $(\mu \mathrm{M})$} & $18^{\circ} \mathrm{C}$ & $88.4 \pm 22.6 \mathrm{a}$ & $106.4 \pm 20.8 a b$ & $90.7 \pm 26.1 \mathrm{a}$ & $105.9 \pm 35.8 b$ & \multirow[t]{2}{*}{$* * *$} & \multirow[t]{2}{*}{ * } & \multirow[t]{2}{*}{ ns } \\
\hline & $25^{\circ} \mathrm{C}$ & $113.9 \pm 13.4 \mathrm{a}$ & $117.3 \pm 19.7 \mathrm{ab}$ & $106.4 \pm 17.1 \mathrm{a}$ & $139.1 \pm 23.5 b$ & & & \\
\hline \multirow[t]{2}{*}{ Anti-protease Activity (\%) } & $18^{\circ} \mathrm{C}$ & $76.5 \pm 8.5$ & $76.4 \pm 7.1$ & $76.6 \pm 6.7$ & $77.5 \pm 5.1$ & \multirow[t]{2}{*}{ ns } & \multirow[t]{2}{*}{ ns } & \multirow[t]{2}{*}{ ns } \\
\hline & $25^{\circ} \mathrm{C}$ & $79.4 \pm 8.2$ & $77.6 \pm 7.1$ & $77.6 \pm 9.5$ & $77.1 \pm 5.0$ & & & \\
\hline \multirow[t]{2}{*}{ Total Immunoglobulin $\left(\mathrm{mg} \mathrm{ml}^{-1}\right)$} & $18^{\circ} \mathrm{C}$ & $18.8 \pm 1.6$ & $20.0 \pm 3.1$ & $19.3 \pm 2.8$ & $20.1 \pm 2.2$ & \multirow[t]{2}{*}{$* * *$} & \multirow[t]{2}{*}{ ns } & \multirow[t]{2}{*}{$*$} \\
\hline & $25^{\circ} \mathrm{C}$ & $18.3 \pm 1.5 b$ & $17.2 \pm 1.8 \mathrm{ab}$ & $18.0 \pm 3.0 \mathrm{~b}$ & $15.2 \pm 2.0 \mathrm{a}$ & & & \\
\hline
\end{tabular}

Values presented as means \pm standard deviation $(\mathrm{n}=9)$.

${ }^{*} \mathrm{P}<0.05$; ${ }^{* * *} \mathrm{P}<0.001$; ns: not significant.

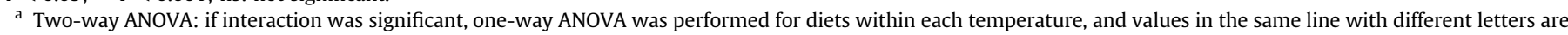
significantly different $(P<0.05)$. 


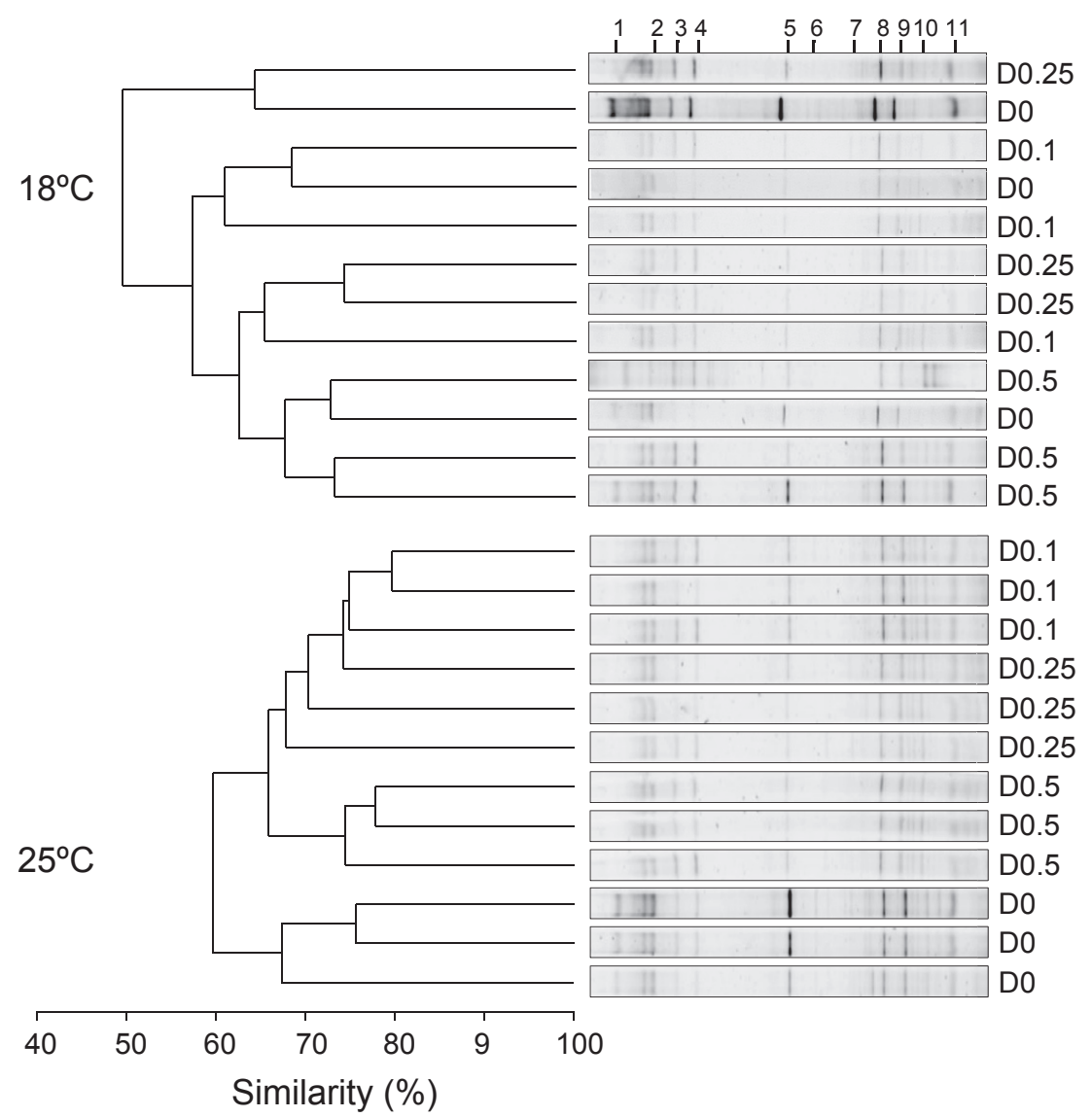

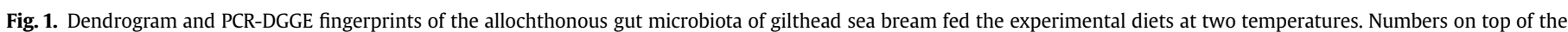
figure (1-11) indicate bands excised for sequence analysis.

Table 6

Ecological parameters obtained from PCR-DGGE fingerprints of gut allochthonous microbiota of gilthead sea bream fed the experimental diets at two temperatures.

\begin{tabular}{|c|c|c|c|c|c|c|c|c|}
\hline & \multirow[t]{2}{*}{ Temperature } & \multicolumn{4}{|l|}{ Diets } & \multicolumn{3}{|c|}{ Two-way ANOVA } \\
\hline & & D0 & D0.1 & D0.25 & D0.5 & Temperature & Diets & Interaction \\
\hline \multirow[t]{2}{*}{ OTUs $^{\mathrm{a}}$} & $18^{\circ} \mathrm{C}$ & $27.3 \pm 4.5$ & $23.3 \pm 4.0$ & $26.7 \pm 6.0$ & $28.0 \pm 2.0$ & ns & ns & ns \\
\hline & $25^{\circ} \mathrm{C}$ & $32.0 \pm 3.5$ & $28.0 \pm 2.0$ & $25.3 \pm 2.5$ & $25.3 \pm 3.1$ & & & \\
\hline \multirow[t]{2}{*}{ Richness $^{\text {b }}$} & $18^{\circ} \mathrm{C}$ & $1.55 \pm 0.22$ & $1.35 \pm 0.23$ & $1.53 \pm 0.32$ & $1.60 \pm 0.11$ & ns & ns & ns \\
\hline & $25^{\circ} \mathrm{C}$ & $1.82 \pm 0.20$ & $1.61 \pm 0.12$ & $1.46 \pm 0.14$ & $1.46 \pm 0.18$ & & & \\
\hline \multirow[t]{2}{*}{ Diversity $^{c}$} & $18^{\circ} \mathrm{C}$ & $3.27 \pm 0.14$ & $3.11 \pm 0.16$ & $3.23 \pm 0.21$ & $3.29 \pm 0.06$ & ns & ns & ns \\
\hline & $25^{\circ} \mathrm{C}$ & $3.43 \pm 0.10$ & $3.30 \pm 0.07$ & $3.21 \pm 0.09$ & $3.20 \pm 0.12$ & & & \\
\hline \multirow[t]{2}{*}{ SIMPER Similarity (\%) } & $18^{\circ} \mathrm{C}$ & $53.2 \pm 12.9$ & $57.7 \pm 4.1$ & $55.0 \pm 16.7$ & $70.2 \pm 2.6$ & $* *$ & ns & ns \\
\hline & $25^{\circ} \mathrm{C}$ & $70.1 \pm 5.0$ & $76.5 \pm 2.9$ & $69.1 \pm 1.8$ & $75.5 \pm 3.0$ & & & \\
\hline
\end{tabular}

Values presented as means \pm standard deviation ( $\mathrm{n}=3$ per treatment pooled from 6 fish).

${ }^{* *} \mathrm{P}<0.01$; ns: not significant.

a OTUs: average number of operational taxonomic units.

b Margalef species richness: $d=(S-1) / \log (N)$.

c Shannons diversity index: $\mathrm{H}^{\prime}=-\sum(\mathrm{pi}(\operatorname{lnpi}))$.

d SIMPER: similarity percentage within group replicates.

immune system affect host health [15,16]. Still, most studies on prebiotics effects in fish performance and immune status did not evaluate gut microbiota [18-23]. Results of the present study failed to show any interaction between gut microbiota composition and fish immune status in relation to dietary prebiotics.

The hematological profile was also almost unaffected by dietary scFOS. In fish, studies on prebiotic effects on differential WBC counts reported either lack of effects [24,25] or increased lymphocytes number [53]. On the contrary, in the present study, fish fed the $0.1 \%$ scFOS diet had a lower number of circulating lymphocytes, but this effect was only observed at $25^{\circ} \mathrm{C}$. In fish fed the scFOS, but not the control diet, temperature also affected lymphocytes numbers, which were higher in fish reared at $18{ }^{\circ} \mathrm{C}$. This evidences the importance of rearing temperature in prebiotics action mode and deserves to be further investigated.

Prebiotics fermentation by gut bacteria produces short chain fatty acids (SCFAs) such as propionic, acetic, and butyric acid. Moreover, different gut bacteria lead to the production of different proportions of SCFAs [54]. SCFAs such as butyric acid are known to have immunomodulatory effects, and butyric acid was already 
Table 7

Identified bacterial species from the DNA sequencing of the allochthonous gut communities of gilthead sea bream fed the experimental diets at two temperatures.

\begin{tabular}{|c|c|c|c|}
\hline Band & Nearest neighbor & $\begin{array}{l}\text { Similarity to nearest } \\
\text { neighbor }\end{array}$ & $\begin{array}{l}\text { Accession number of nearest } \\
\text { neighbor }\end{array}$ \\
\hline 1 & Uncultured bacteria from fermented foods such as pearl millet slurries & 84 & FN775030.1 \\
\hline 2 & Uncultured bacterium isolate DGGE gel band from the jejunum, ileum and cecum of weaned piglets & 95 & JX183818.1 \\
\hline 3 & Uncultured bacterium clone from Antarctic soil & 86 & HM710432.1 \\
\hline 4 & Lactobacillus aviarius & 97 & M58808.2 \\
\hline 5 & Uncultured bacterium clone from wild and domesticated adult black tiger shrimp (Penaeus monodon) & 93 & KF337424.1 \\
\hline 6 & Uncultured bacterium clone from hybrid tilapia (Tilapia nilotica $\times$ T. aurea) & 90 & EF599660.1 \\
\hline 7 & Uncultured Cyanothece sp. clone from rhizosphere of Arachi hypogaea & 87 & JX628832.1 \\
\hline 8 & Oceanobacillus sp. & 94 & KF958190.1 \\
\hline 9 & Uncultured bacterium clone from Canada goose feces & 97 & FJ390662.1 \\
\hline 10 & Uncultured bacterium isolate DGGE gel band from the jejunum, ileum and cecum of weaned piglets & 99 & JX183818.1 \\
\hline 11 & $\begin{array}{l}\text { Uncultured bacterium clone from subsurface saline soil in Sanhu region of Qaidam Basin on Tibetan } \\
\text { Plateau, China }\end{array}$ & 90 & GU212441.1 \\
\hline
\end{tabular}

Table 8

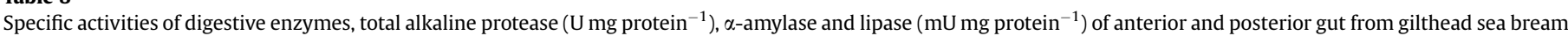
fed the experimental diets at two temperatures.

\begin{tabular}{|c|c|c|c|c|c|c|c|c|}
\hline & \multirow[t]{2}{*}{ Temperature } & \multicolumn{4}{|l|}{ Diets } & \multicolumn{3}{|c|}{ Two-way ANOVA } \\
\hline & & D0 & D0.1 & D0.25 & D0.5 & Temperature & Diets & Interaction \\
\hline \multicolumn{9}{|l|}{ Anterior gut } \\
\hline \multirow[t]{2}{*}{ Total Alkaline Protease } & $18^{\circ} \mathrm{C}$ & $353.0 \pm 75.8$ & $284.4 \pm 108.2$ & $274.4 \pm 79.5$ & $364.3 \pm 89.2$ & ns & ns & ns \\
\hline & $25^{\circ} \mathrm{C}$ & $371.4 \pm 112.1$ & $332.0 \pm 125.5$ & $303.0 \pm 163.3$ & $269.3 \pm 87.3$ & & & \\
\hline \multirow[t]{2}{*}{$\alpha$-Amylase } & $18{ }^{\circ} \mathrm{C}$ & $269.9 \pm 128.3$ & $254.1 \pm 91.3$ & $225.3 \pm 127.9$ & $210.4 \pm 96.6$ & $* * *$ & ns & ns \\
\hline & $25^{\circ} \mathrm{C}$ & $804.9 \pm 267.3$ & $693.4 \pm 426.7$ & $507.7 \pm 193.3$ & $606.0 \pm 235.8$ & & & \\
\hline \multirow[t]{2}{*}{ Lipase } & $18^{\circ} \mathrm{C}$ & $14.5 \pm 4.1$ & $14.0 \pm 2.3$ & $12.3 \pm 3.0$ & $13.6 \pm 5.6$ & $*$ & ns & ns \\
\hline & $25^{\circ} \mathrm{C}$ & $21.0 \pm 5.9$ & $16.9 \pm 9.0$ & $14.9 \pm 9.0$ & $15.8 \pm 5.7$ & & & \\
\hline \multicolumn{9}{|l|}{ Posterior gut } \\
\hline \multirow[t]{2}{*}{ Total Alkaline Protease } & $18^{\circ} \mathrm{C}$ & $337.1 \pm 101.3 \mathrm{ab}$ & $405.9 \pm 113.9 b$ & $302.1 \pm 162.4 \mathrm{ab}$ & $208.2 \pm 100.6 a$ & $* * *$ & ns & $*$ \\
\hline & $25^{\circ} \mathrm{C}$ & $641.4 \pm 166.8$ & $543.5 \pm 137.6$ & $688.5 \pm 129.7$ & $603.6 \pm 154.4$ & & & \\
\hline \multirow[t]{2}{*}{$\alpha$-Amylase } & $18^{\circ} \mathrm{C}$ & $293.8 \pm 50.1$ & $314.4 \pm 115.0$ & $222.7 \pm 71.1$ & $161.5 \pm 83.7$ & $* * *$ & ns & ns \\
\hline & $25^{\circ} \mathrm{C}$ & $1009.7 \pm 262.3$ & $910.6 \pm 204.2$ & $1011.3 \pm 74.9$ & $932.1 \pm 240.5$ & & & \\
\hline \multirow[t]{2}{*}{ Lipase } & $18^{\circ} \mathrm{C}$ & $15.6 \pm 6.4$ & $16.3 \pm 6.2$ & $15.9 \pm 10.1$ & $13.4 \pm 5.6$ & $* * *$ & ns & ns \\
\hline & $25^{\circ} \mathrm{C}$ & $33.5 \pm 7.7$ & $24.9 \pm 5.0$ & $27.0 \pm 4.1$ & $30.1 \pm 4.3$ & & & \\
\hline
\end{tabular}

Values presented as means \pm standard deviation $(\mathrm{n}=9)$.

${ }^{*} \mathrm{P}<0.05$; ${ }^{* * *} \mathrm{P}<0.001$; ns: not significant.

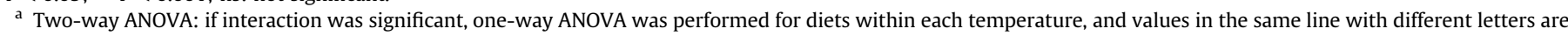
significantly different $(\mathrm{P}<0.05)$.

Table 9

Gut histology of gilthead sea bream fed the experimental diets at two temperatures.

\begin{tabular}{|c|c|c|c|c|c|}
\hline & \multirow[t]{2}{*}{ Temperature } & \multicolumn{4}{|l|}{ Diets } \\
\hline & & D0 & D0.1 & D0.25 & D0.5 \\
\hline \multirow[t]{2}{*}{ Anterior gut } & $18^{\circ} \mathrm{C}$ & $1.76 \pm 0.15$ & $1.76 \pm 0.17$ & $1.73 \pm 0.15$ & $1.73 \pm 0.16$ \\
\hline & $25^{\circ} \mathrm{C}$ & $1.71 \pm 0.17$ & $1.69 \pm 0.14$ & $1.86 \pm 0.16$ & $1.86 \pm 0.15$ \\
\hline \multirow[t]{2}{*}{ Posterior gut } & $18^{\circ} \mathrm{C}$ & $1.86 \pm 0.47$ & $1.85 \pm 0.18$ & $1.90 \pm 0.20$ & $1.81 \pm 0.24$ \\
\hline & $25^{\circ} \mathrm{C}$ & $1.81 \pm 0.12$ & $1.72 \pm 0.27$ & $1.77 \pm 0.25$ & $1.84 \pm 0.17$ \\
\hline
\end{tabular}

Values presented as means \pm standard deviation $(n=9)$. Score from 1 to 5 , with 5 indicating major alterations.

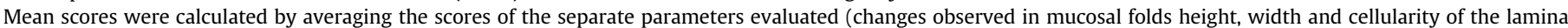
propria and submucosa, number of intraepithelial lymphocytes, nucleus position within the enterocytes and size and variation of enterocyte vacuolization).

reported to suppress lymphocytes proliferation [55]. In beluga (Huso huso), lymphocytes percentage was reduced in fish fed a $0.2 \%$ MOS diet [56]. Thus, the reduction of lymphocytes numbers observed in the present study may be related to an increased production of butyric acid in fish fed diets with $0.1 \%$ scFOS, but this requires to be confirmed.

Fish reared at $25{ }^{\circ} \mathrm{C}$ exhibited higher $\mathrm{Hb}$ content and $\mathrm{RBC}$ numbers compared to fish reared at $18^{\circ} \mathrm{C}$. This was not unexpected, since $\mathrm{RBC}$ are responsible for providing $\mathrm{O}_{2}$ to the cells, and fish $\mathrm{O}_{2}$ requirement increases while $\mathrm{O}_{2}$ dissolved in water decreases with the increase of water temperature. Similar results were previously reported for rainbow trout (Oncorhynchus mykiss) exposed to simulated winter, spring, and summer conditions [57].
Plasma NO values are in line with those of RBC. Indeed, besides its immune function, $\mathrm{NO}$ also acts as a vasodilator, being produced at higher levels in fish reared at higher temperatures [58]. NO production was also affected by prebiotic incorporation, with higher values being observed in fish fed the $0.5 \%$ scFOS diet. In turbot, it was reported that Lactococcus lactis increased NO production in head kidney macrophage and serum, both in vitro and in vivo, respectively, possibly due to a synergistic effect between L. lactis and cytokines or other soluble factors [59]. Although higher LAB population in fish fed the $0.5 \%$ scFOS diet cannot be discarded, we were unable to detect it by the PCR-DGGE technique used in the present study.

At $25{ }^{\circ} \mathrm{C}$, plasma total Ig was lower in fish fed the $0.5 \%$ scFOS 
diet. Although, such a result was not observed on lymphocytes numbers, B-cells the ones responsible for Ig secretion [41,60], might change the Ig production rates, without changing total cell numbers. Regarding temperature effect, total Ig results are in line with lymphocytes results, being higher in fish reared at $18^{\circ} \mathrm{C}$, with fish fed control diet being not affected by the rearing temperature.

Though it is usually assumed that adaptive immune parameters tend to be suppressed at low temperatures [41], this is not a general rule. Indeed, in this study, fish reared at $18{ }^{\circ} \mathrm{C}$ and fed scFOS had higher total Ig values and lymphocyte counts than fish reared at $25{ }^{\circ} \mathrm{C}$.

Monocytes and neutrophils are known to have strong phagocytosis ability and bactericidal and anti-protease activities [61,62]. In this study, monocytes and neutrophils numbers were higher in fish reared at $18{ }^{\circ} \mathrm{C}$, but plasma bactericidal activity and NO production were higher in fish reared at $25{ }^{\circ} \mathrm{C}$. In contrast, antiprotease activity was unaffected by water temperature. As in this study, Machado et al. [47] also observed that neutrophils and monocytes numbers may increase without showing increased humoral immune parameters, such as lysozyme and peroxidase activities.

In the present study, an effect of prebiotic incorporation on gut bacterial composition could not be detected. This contradicts results of other studies that reported FOS effects in growth and survival of beneficial bacteria present in fish gut $[24,25,63]$. Although PCR-DGGE is a useful technique to assess microbial community structures and ecological characteristics, allowing identification of bacteria that are not detected using culture dependent techniques [64], it is not as sensible as quantitative techniques such as FISH, qPCR, and next-generation sequencing. These quantitative techniques should therefore be preferred in future studies to overcome the semi-quantitative limitations associated with PCR-DGGE $[64,65]$. The apparent lack of scFOS effect could also be related with the high levels of fiber and oligosaccharides already present in the plant feedstuffs used in the experimental diets. For instance, soybean meal contains $4-5 \%$ oligosaccharides which may also have prebiotic-like properties, thus masking the effect of scFOS. Accordingly, also in gilthead sea bream, an absence of gut microbiota modulation was reported in fish fed a soy-rich diet supplemented with MOS, contrasting with gut microbiota modulation by MOS when fish were fed fish-meal-based diets [36].

Although several studies reported changes in gut microbiota communities in response to water temperature [12,39,66,67], such effect was not observed in the present study. The only detectable effect was an increase of similarity between DGGE profiles at $25^{\circ} \mathrm{C}$, indicating that higher temperatures might modulate gut bacteria population towards a higher similarity between samples. To the authors' knowledge there is only one study on the effects of temperature on prebiotics on fish gut microbiota modulation [12]. In contrast with present results, in that study it was shown that turbot fed scFOS presented higher gut bacteria richness and diversity at $20{ }^{\circ} \mathrm{C}$ than at $15^{\circ} \mathrm{C}$. There are other studies on the effects of temperature on fish gut microbiota, but used culture-dependent methods and are therefore difficult to compare with the cultureindependent results presented in this study [39,66,67].

Regarding OTUs identity, the bacteria identified in the present study corresponded mostly to uncultured bacteria. Similar results were also observed in previous studies $[8,27,68]$. This is not surprising, since it is known that cultivable bacteria correspond to a small fraction of the total bacterial diversity that can be found associated with a complex and rich environment like the gut $[68,69]$. Nevertheless, a Lactobacillus sp., a genus of the LAB clade, was found among our samples. Although not dominant, LAB bacteria are often isolated from the gut of several fish species $[39,70-72]$ and, interestingly, FOS was reported as supporting their growth and survival. The other two genus identified (Cyanothece and Oceanobacillus) corresponded to bacteria commonly found associated with marine environments $[73,74]$.

The higher digestive enzymatic activity observed in fish reared at $25{ }^{\circ} \mathrm{C}$ correlates well with the higher feed intake, feed efficiency, and growth performance observed, and reported in Guerreiro et al. [11]. Although several studies reported increased digestive enzymatic activity or increased apparent nutrient digestibility in fish fed prebiotics $[30-33,75,76]$, in the present study scFOS did not affect digestive enzymatic activity. This is in agreement with the lack of detectable scFOS effects in gut microbiota, as increased digestive enzymes activities were also suggested to be related to changes in gut microbiota. As in this study, other studies also failed to show increased digestive enzymatic activity in fish fed prebiotics supplemented diets [12,77-79]. Only TAP activity in the posterior gut was affected by temperature, however depending on fish diet, which reinforces the potential effect of temperature in prebiotic action.

Prebiotics, namely FOS, galactooligosaccharides (GOS), and MOS, have been reported to increase gut absorptive surface area due to increased microvilli density and height [26,35-37]. In the present study, however, no changes in gut histomorphology were noticed. This may also be related with the level of soybean meal in the experimental diets used in this study. Indeed, Bonaldo et al. [80] were able to observe minor histological alterations in the distal gut of gilthead sea bream fed diets including soybean meal at $30 \%$ but not at lower level (18\%). Hence, whether deleterious effects of a more challenging diet could be alleviated by scFOS should be addressed in future studies. Also in gilthead sea bream, Dimitroglou et al. [36] found that dietary MOS had no effect on anterior gut mucosal folds morphology when observed by light microscopy. However, using electron microscopy it was shown that MOS positively affected gut ultrastructure. Since in the current study gut ultrastructure was not assessed, changes in gut morphology at ultrastructure level cannot also be discarded.

Results of this study indicate that gilthead sea bream innate immunity appears to be affected by rearing temperature, with particularly higher levels of circulating leucocytes at $18{ }^{\circ} \mathrm{C}$, comparatively to $25^{\circ} \mathrm{C}$. On the contrary, digestive enzymes activities and gut microbiota similarity were higher in fish reared at $25{ }^{\circ} \mathrm{C}$. Overall, dietary scFOS at the tested levels seem to have no major effects on gut microbiota composition, digestive enzymes activities, gut histomorphology and innate immune parameters. Thus, based on present data, it does not seem worthy of including scFOS in gilthead sea bream diets, at least at the tested levels. However, taking into account both the data of this study and that of Guerreiro et al. [11], it seems that rearing temperature might affect prebiotic outcomes, and this should be further exploited in other studies.

\section{Acknowledgments}

This research was partially supported by the European Regional Development Fund (ERDF) through the COMPETE - Operational Competitiveness Programme and national funds through FCT Foundation for Science and Technology, under the projects "PEst-C/ MAR/LA0015/2013 and UID/Multi/04423/2013”. I. Guerreiro, P. Enes, A. Couto and B. Costas were supported by grants (SFRH/BD/ 76139/2011; BPD/39688/2007, BD/47495/2008 and BPD/77210/ 2011, respectively) from FCT, Portugal. C. Serra and A. Salvador were recipient of grants within the Project AQUAIMPROV (reference NORTE-07-0124-FEDER-000038) and Project BioEnv (REF. NORTE07-0124-FEDER-000048), co-financed by the North Portugal Regional Operational Programme (ON.2 - O Novo Norte). We would like to express our thanks to Pedro Correia for the assistance 
during the growth trial. Authors would also thank to Jefo Speciesspecific additives France, for providing the prebiotic.

\section{References}

[1] M.B. Roberfroid, Introducing inulin-type fructans, Br. J. Nutr. 93 (2005) S13-S25.

[2] S.K. Song, B.R. Beck, D. Kim, J. Park, J. Kim, H.D. Kim, E. Ringø, Prebiotics as immunostimulants in aquaculture: a review, Fish Shellfish Immunol. 40 (2014) 40-48.

[3] E. Ringø, R.E. Olsen, T.Ø. Gifstad, R.A. Dalmo, H. Amlund, G.I. Hemre, A.M. Bakke, Prebiotics in aquaculture: a review, Aquac. Nutr. 16 (2010) $117-136$.

[4] E. Ringø, A. Dimitroglou, S.H. Hoseinifar, S.J. Davies, Prebiotics in finfish: an update, in: D.L. Merrifield, E. Ringø (Eds.), Aquaculture Nutrition: Gut Health, Probiotics and Prebiotics, John Wiley \& Sons Ltd., Chichester, UK, 2014, pp. $360-400$.

[5] A. Dimitroglou, D.L. Merrifield, O. Carnevali, S. Picchietti, M. Avella, C. Daniels, D. Güroy, S.J. Davies, Microbial manipulations to improve fish health and production - A Mediterranean perspective, Fish Shellfish Immunol. 30 (2011a) 1-16.

[6] F.R.J. Bornet, F. Brouns, Y. Tashiro, V. Duvillier, Nutritional aspects of shortchain fructooligosaccharides: natural occurrence, chemistry, physiology and health implications, Dig. Liver Dis. 34 (2002) S111-S120.

[7] L.V. Hui-yuan, Z. Zhi-gang, F. Rudeaux, F. Respondek, Effects of dietary short chain fructo-oligosaccharides on intestinal microflora, mortality and growth performance of Oreochromis aureus ơ $\times$ O. niloticus + , Chin. J. Anim. Nutr. 19 (2007) $1-6$.

[8] Z.-G. Zhou, S. He, Y. Liu, P. Shi, G. Huang, B. Yao, The effects of dietary yeast culture or short-chain fructo-oligosaccharides on the intestinal autochthonous bacterial communities in juvenile hybrid tilapia, Oreochromis niloticus i $\times$ Oreochromis aureus ô, J. World Aquac. Soc. 40 (2009) 450-459.

[9] I. Guerreiro, A. Pérez-Jiménez, B. Costas, A. Oliva-Teles, Effect of temperature and short chain fructooligosaccharides supplementation on the hepatic oxidative status and immune response of turbot (Scophthalmus maximus), Fish Shellfish Immunol. 40 (2014) 570-576.

[10] I. Guerreiro, P. Enes, D. Merrifield, S. Davies, A. Oliva-Teles, Effects of shortchain fructooligosaccharides on growth performance and hepatic intermediary metabolism in turbot (Scophthalmus maximus) reared at winter and summer temperatures, Aquac. Nutr. 21 (2015a) 433-443.

[11] I. Guerreiro, P. Enes, A. Oliva-Teles, Effects of short chain fructooligosaccharides (scFOS) and rearing temperature on growth performance and hepatic intermediary metabolism in gilthead sea bream (Sparus aurata) juveniles, Fish Physiol. Biochem. 41 (2015b) 1333-1344.

[12] I. Guerreiro, P. Enes, A. Rodiles, D. Merrifield, A. Oliva-Teles, Effects of rearing temperature and dietary short-chain fructooligosaccharides supplementation on allochthonous gut microbiota, digestive enzymes activities and intestine health of turbot (Scophthalmus maximus L.) juveniles, Aquac. Nutr. (2015c), http://dx.doi.org/10.1111/anu.12277.

[13] I. Guerreiro, A. Oliva-Teles, P. Enes, Improved glucose and lipid metabolism in European sea bass (Dicentrarchus labrax) fed short-chain fructooligosaccharides and xylooligosaccharides, Aquaculture 441 (2015d) 57-63.

[14] S.H. Hoseinifar, H. Eshaghzadeh, H. Vahabzadeh, N. Peykaran Mana, Modulation of growth performances, survival, digestive enzyme activities and intestinal microbiota in common carp (Cyprinus carpio) larvae using short chain fructooligosaccharide, Aquac. Res. (2015), http://dx.doi.org/10.1111/ are.12777.

[15] T.S. Kemgang, S. Kapila, V.P. Shanmugam, R. Kapila, Cross-talk between probiotic lactobacilli and host immune system, J. Appl. Microbiol. 117 (2014) 303-319.

[16] C.M. Galdeano, G. Perdigón, The probiotic bacterium Lactobacillus casei induces activation of the gut mucosal immune system through innate immunity, Clin. Vaccine Immunol. 13 (2006) 219-226.

[17] L. Vogt, U. Ramasamy, D. Meyer, G. Pullens, K. Venema, M.M. Faas, H.A. Schols, P. de Vos, Immune modulation by different types of $\beta 2 \rightarrow 1$-fructans is toll-like receptor dependent, PLoS One 8 (7) (2013) e68367.

[18] R. Cerezuela, A. Cuesta, J. Meseguer, M.A. Esteban, Effects of inulin on gilthead seabream (Sparus aurata L.) innate immune parameters, Fish Shellfish Immunol. 24 (2008) 663-668.

[19] Y. Li, Y.J. Wang, L. Wang, K.Y. Jiang, Influence of several non-nutrient additives on nonspecific immunity and growth of juvenile turbot, Scophthalmus maximus L, Aquac. Nutr. 14 (2008) 387-395.

[20] Q. Ai, H. Xu, K. Mai, W. Xu, J. Wang, W. Zhang, Effects of dietary supplementation of Bacillus subtilis and fructooligosaccharide on growth performance, survival, non-specific immune response and disease resistance of juvenile large yellow croaker, Larimichthys crocea, Aquaculture 317 (2011) $155-161$.

[21] N. Gültepe, O. Hisar, S. Salnur, B. Hoşsu, T.T. Tanrikul, S. Aydın, Preliminary assessment of dietary mannanoligosaccharides on growth performance and health status of gilthead seabream Sparus auratus, J. Aquat. Anim. Health 24 (2012) 37-42.

[22] C.-N. Zhang, X.-F. Li, W.-N. Xu, G.-Z. Jiang, K.-L. Lu, L.-N. Wang, W.-B. Liu, Combined effects of dietary fructooligosaccharide and Bacillus licheniformis on innate immunity, antioxidant capability and disease resistance of triangular bream (Megalobrama terminalis), Fish Shellfish Immunol. 35 (2013) 1380-1386.

[23] C.-N. Zhang, H.-Y. Tian, X.-F. Li, J. Zhu, D.-S. Cai, C. Xu, F. Wang, D.-D. Zhang, W.-B. Liu, The effects of fructooligosaccharide on the immune response, antioxidant capability and HSP70 and HSP90 expressions in blunt snout bream (Megalobrama amblycephala Yih) under high heat stress, Aquaculture 433 (2014) 458-466.

[24] R. Akrami, Y. Iri, H.K. Rostami, M.R. Mansour, Effect of dietary supplementation of fructooligosaccharide (FOS) on growth performance, survival, lactobacillus bacterial population and hemato-immunological parameters of stellate sturgeon (Acipenser stellatus) juveniles, Fish Shellfish Immunol. 35 (2013a) 1235-1239.

[25] S.H. Hoseinifar, N. Soleimani, E. Ringø, Effects of dietary fructo-oligosaccharide supplementation on the growth performance, haemato-immunological parameters, gut microbiota and stress resistance of common carp (Cyprinus carpio) fry, Br. J. Nutr. 112 (2014) 1296-1302.

[26] Q. Zhou, J.A. Buentello, D.M. Gatlin III, Effects of dietary prebiotics on growth performance, immune response and intestinal morphology of red drum (Sciaenops ocellatus), Aquaculture 309 (2010) 253-257.

[27] P. Li, G.S. Burr, D.M. Gatlin III, M.E. Hume, S. Patnaik, F.L. Castille, A.L. Lawrence, Dietary supplementation of short-chain fructooligosaccharides influences gastrointestinal microbiota composition and immunity characteristics of Pacific white shrimp, Litopenaeus vannamei, cultured in a recirculating system, J. Nutr. 137 (2007) 2763-2768.

[28] R. Cerezuela, F.A. Guardiola, J. Meseguer, M.Á. Esteban, Increases in immune parameters by inulin and Bacillus subtilis dietary administration to gilthead sea bream (Sparus aurata L.) did not correlate with disease resistance to Photobacterium damselae, Fish Shellfish Immunol. 32 (2012) 1032-1040.

[29] R. Cerezuela, M. Fumanal, S.T. Tapia-Paniagua, J. Meseguer, M.A. Morinigo, M.A. Esteban, Changes in intestinal morphology and microbiota caused by dietary administration of inulin and Bacillus subtilis in gilthead sea bream (Sparus aurata L.) specimens, Fish Shellfish Immunol. 34 (2013) 1063-1070.

[30] L. Renjie, S. Shidi, Z. Bangiie, The effect of fructo-oligosaccharides on blood RBC count and digestive enzyme activities of Oxyeleotris lineolatus, Afr. J. Microbiol. Res. 4 (2010) 1909-1913.

[31] N. Soleimani, S.H. Hoseinifar, D.L. Merrifield, M. Barati, Z.H. Abadi, Dietary supplementation of fructooligosaccharide (FOS) improves the innate immune response, stress resistance, digestive enzyme activities and growth performance of Caspian roach (Rutilus rutilus) fry, Fish Shellfish Immunol. 32 (2012) $316-321$.

[32] Y. Wu, W.-B. Liu, H.-Y. Li, W.-N. Xu, J.-X. He, X.-F. Li, G.-Z. Jiang, Effects of dietary supplementation of fructooligosaccharide on growth performance body composition, intestinal enzymes activities and histology of blunt snout bream (Megalobrama amblycephala) fingerlings, Aquac. Nutr. 19 (2013) $886-894$.

[33] N. Gültepe, S. Salnur, B. Hoșsu, O. Hisar, Dietary supplementation with mannanoligosaccharides (MOS) from Bio-Mos enhances growth parameters and digestive capacity of gilthead sea bream (Sparus aurata), Aquac. Nutr. 17 (2011) 482-487.

[34] M.A. Genc, E. Yilmaz, E. Genc, M. Aktas, Effects of dietary mannan oligosaccharides (MOS) on growth, body composition, and intestine and liver histology of the hybrid tilapia (Oreochromis niloticus x O. aureus), Isr. J. Aquac. Bamidgeh. 59 (2007) 10-16.

[35] A. Dimitroglou, D.L. Merrifield, R. Moate, S.J. Davies, P. Spring, J. Sweetman, G. Bradley, Dietary mannan oligosaccharide supplementation modulates intestinal microbial ecology and improves gut morphology of rainbow trout, Oncorhynchus mykiss (Walbaum), J. Anim. Sci. 87 (2009) 3226-3234.

[36] A. Dimitroglou, D.L. Merrifield, P. Spring, J. Sweetman, R. Moate, S.J. Davies, Effects of mannan oligosaccharide (MOS) supplementation on growth performance, feed utilisation, intestinal histology and gut microbiota of gilthead sea bream (Sparus aurata), Aquaculture 300 (2010) 182-188.

[37] A. Dimitroglou, R. Moate, T. Janssens, P. Spring, J.W. Sweetman, J.D. Davies, Field observations on the effect of a mannan oligosaccharide on mortality and intestinal integrity of sole (Solea senegalensis, Kaup) infected by Photobacterium damselae subsp. piscicida, J. Aquac. Res. Dev. (S1:013) (2011b) http://dx.doi.org/10.4172/2155-9546.S1-013.

[38] A. Dimitroglou, P. Reynolds, B. Ravnoy, F. Johnsen, J.W. Sweetman, J. Johansen, S.J. Davies, The effect of mannan oligosaccharide supplementation on Atlantic salmon smolts (Salmo salar L.) fed diets with high levels of plant proteins, J. Aquac. Res. Dev. S1:011 (2011c), http://dx.doi.org/10.4172/2155-9546.S1011.

[39] T. Hagi, D. Tanka, Y. Iwamura, T. Hoshino, Diversity and seasonal changes in lactic acid bacteria in the intestinal tract of cultured freshwater fish, Aquaculture 234 (2004) 335-346.

[40] S. Denev, Y. Staykov, R. Moutafchieva, G. Beev, Microbial ecology of the gastrointestinal tract of fish and the potential application of probiotics and prebiotics in finfish aquaculture, Int. Aquat. Res. 1 (2009) 1-29.

[41] B. Magnadottir, Immunological control of fish diseases, Mar. Biotechnol. 12 (2010) 361-379.

[42] C. Alexander, N.P. Sahu, A.K. Pal, M.S. Akhtar, Haemato-immunological and stress responses of Labeo rohita (Hamilton) fingerlings: effect of rearing temperature and dietary gelatinized carbohydrate, J. Anim. Physiol. Anim. Nutr. 95 (2011) 653-663.

[43] S. Torrecillas, D. Montero, M.J. Caballero, L. Robaina, M.J. Zamorano 
J. Sweetman, M. Izquierdo, Effects of dietary concentrated mannan oligosaccharides supplementation on growth, gut mucosal immune system and liver lipid metabolism of European sea bass (Dicentrarchus labrax) juveniles, Fish Shellfish Immunol. 42 (2015) 508-516.

[44] G.R. Gibson, M.B. Roberfroid, Dietary modulation of the human colonie microbiota: introducing the concept of prebiotics, J. Nutr. 125 (1995) 1401-1412.

[45] AOAC, Official Methods of Analysis, Association of Official Analytical Chemists Gaithersburg, Maryland, USA., 2000, 1018 p.

[46] H.O. Beutler, Starch, in: H.U. Bergmeyer (Ed.), Methods of Enzymatic Analysis, vol. 6, Verlag Chemie, Weinheim, Basel, 1984, pp. 2-10.

[47] M. Machado, R. Azeredo, P. Díaz-Rosales, A. Afonso, H. Peres, A. Oliva-Teles B. Costas, Dietary tryptophan and methionine as modulators of European seabass (Dicentrarchus labrax) immune status and inflammatory response Fish Shellfish Immunol. 42 (2015) 353-362.

[48] D.G. Pitcher, N.A. Saunders, R.J. Owen, Rapid extraction of bacterial genomic DNA with guanidium thiocyanate, Lett. Appl. Microbiol. 8 (1989) 151-156.

[49] G. Muyzer, E.C. de Waal, A.G. Uitterlinden, Profiling of complex microbial populations by denaturing gradient gel electrophoresis analysis of polymerase chain reaction-amplified genes coding for 16S rRNA, Appl. Environ. Microbiol. 59 (1993) 695-700

[50] A. Couto, T.M. Kortner, M. Penn, G. Østby, A.M. Bakke, Å. Krogdahl, A. OlivaTeles, Saponins and phytosterols in diets for European sea bass (Dicentrarchus labrax) juveniles: effects on growth, intestinal morphology and physiology, Aquac. Nutr. 21 (2015) 180-193.

[51] Å. Krogdahl, A.M. Bakke-McKellep, G. Baeverfjord, Effects of graded levels of standard soybean meal on intestinal structure, mucosal enzyme activities, and pancreatic response in Atlantic salmon (Salmo salar L.), Aquac. Nutr. 9 (2003) $361-371$.

[52] M.H. Penn, E.Å. Bendiksen, P. Campbell, Å. Krogdahl, High dietary level of pe protein concentrate induces intestinal enteropathy in Atlantic salmon (Salmo salar L.), Aquaculture 310 (2011) 267-273.

[53] S.H. Hoseinifar, A. Mirvaghefi, D.L. Merrifield, B.M. Amiri, S. Yelghi, K.D. Bastami, The study of some haematological and serum biochemical parameters of juvenile beluga (Huso huso) fed oligofructose, Fish Physiol. Biochem. 37 (2011b) 91-96.

[54] J.E. Teitelbaum, Prebiotics and lipid metabolism, in: S.S. Cho, T. Finocchiaro (Eds.), Handbook of Prebiotics and Probiotics Ingredients: Health Benefits and Food Applications, CRC Press, USA, 2009, pp. 209-220.

[55] G. Tzortzis, J. Vulevic, Galacto-oligosaccharide prebiotics, in: D. Charalampopoulos, R.A. Rastall (Eds.), Prebiotics and Probiotics Science and Technology, Springer Science+Business Media, LLC, New York, USA, 2009, pp. 207-245.

[56] R. Akrami, M.R. Mansour, Sh Ghobadi, E. Ahmadifar, M.S. Khoshroudi, M.S.M. Haji, Effect of prebiotic mannan oligosaccharide on hematological and blood serum biochemical parameters of cultured juvenile great sturgeon (Huso huso Linnaeus, 1754), J. Appl. Ichthyol. 29 (2013b) 1214-1218.

[57] A.H. Houston, N. Dobric, R. Kahurananga, The nature of hematological response in fish - Studies on rainbow trout Oncorhynchus mykiss exposed to simulated winter, spring and summer conditions, Fish Physiol. Biochem. 15 (1996) 339-347.

[58] A.M. Rieger, D.R. Barreda, Antimicrobial mechanisms of fish leukocytes, Dev. Comp. Immunol. 35 (2011) 1238-1245.

[59] L. Villamil, C. Tafalla, A. Figueras, B. Novoa, Evaluation of immunomodulatory effects of lactic acid bacteria in turbot (Scophthalmus maximus), Clin. Diagn. Lab. Immunol. 9 (2002) 1318-1323.

[60] L. Borghesi, C. Milcarek, From B cell to plasma cell regulation of V(D). recombination and antibody secretion, Immunol. Res, 36 (2006) 27-32.

[61] C.J. Secombes, A.E. Ellis, The Immunology of Teleosts, in: R.J. Roberts (Ed.), Fish Pathology, John Wiley \& Sons, Ltd, Chichester, UK, 2012, pp. 144-167.

[62] R. Castro, C. Tafalla, Overview of fish immunity, in: B.H. Beck, E. Peatman (Eds.) Mucosal Health in Aquaculture Academic Press, USA, 2015, pp. 3-55.

[63] S.H. Hoseinifar, A. Mirvaghefi, B.M. Amiri, H.K. Rostami, D.L. Merrifield, The effects of oligofructose on growth performance, survival and autochthonous intestinal microbiota of beluga (Huso huso) juveniles, Aquac. Nutr. 17 (2011a) 498-504.

[64] Z. Zhou, B. Yao, J. Romero, P. Waines, E. Ringø, M. Emery, M.R. Liles, D.L. Merrifield, Methodological approaches used to assess fish gastrointestinal communities, in: D.L. Merrifield, E. Ringø (Eds.), Aquaculture Nutrition: Gut Health, Probiotics and Prebiotics, John Wiley \& Sons Ltd., Chichester, UK, 2014, pp. 101-127.

[65] G. Rastogi, R.K. Sani, Molecular techniques to assess microbial community structure, function, and dynamics in the environment, in: I. Ahmad, F. Ahmad, J. Pichtel (Eds.), Microbes and Microbial Technology: Agricultural and Environmental Applications, Springer, New York, NY, 2011, pp. 29-57.

[66] A.H. Al-Harbi, M.N. Uddin, Seasonal variation in the intestinal bacterial flora of hybrid tilapia (Oreochromis niloticus $\times$ Oreochromis aureus) cultured in earthen ponds in Saudi Arabia, Aquaculture 229 (2004) 37-44.

[67] A. Bucio, R. Hartemink, J.W. Schrama, J. Verreth, F.M. Rombouts, Presence of lactobacilli in the intestinal content of freshwater fish from a river and from a farm with a recirculation system, Food Microbiol. 23 (2006) 476-482.

[68] D.L. Merrifield, D. Güroy, B. Güroy, M.J. Emery, C.A. Llewellyn, S. Skill, S.J. Davies, Assessment of Chlorogloeopsis as a novel microbial dietary supplement for red tilapia (Oreochromis niloticus), Aquaculture 299 (2010) $128-133$.

[69] E.J. Steward, Growing unculturable bacteria, J. Bacteriol. 194 (2012) $4151-4160$

[70] E. Ringø, F.J. Gatesoupe, Lactic acid bacteria in fish: a review, Aquaculture 160 (1998) 177-203.

[71] F. Askarian, A. Kousha, E. Ringø, Isolation of lactic acid bacteria from the gastrointestinal tracts of beluga (Huso huso) and Persian sturgeon (Acipenser persicus), J. Appl. Ichthyol. 25 (2009) 91-94.

[72] D.L. Merrifield, J.L. Balcázar, C. Daniels, Z. Zhou, O. Carnevali, Y.-Z. Sun, S.H. Hoseinifar, E. Ringø, Indigenous lactic acid bacteria in fish and crustaceans, in: D.L. Merrifield, E. Ringø (Eds.), Aquaculture Nutrition: Gut Health, Probiotics and Prebiotics, John Wiley \& Sons Ltd, Chichester, UK, 2014, pp. $128-168$.

[73] J. Lu, Y. Nogi, H. Takami, Oceanobacillus iheyensis gen. nov., sp. nov., a deep-sea extremely halotolerant and alkaliphilic species isolated from a depth of $1050 \mathrm{~m}$ on the Iheya Ridge, FEMS Microbiol. Lett. 205 (2001) 291-297.

[74] A. Bandyopadhyay, T. Elvitigala, E. Welsh, J. Stöckel, M. Liberton, H. Min, L.A. Sherman, H.B. Pakrasi, Novel metabolic attributes of the genus Cyanothece, comprising a group of unicellular nitrogen-fixing cyanobacteria, mBio 2 (5) (2011) e00214-11.

[75] G. Burr, M. Hume, W.H. Neill, D.M. Gatlin III, Effects of prebiotics on nutrient digestibility of a soybean-meal based diet by red drum Sciaenops ocellatus (Linnaeus), Aquac. Res. 39 (2008) 1680-1686.

[76] B. Xu, Y. Wang, J. Li, Q. Lin, Effect of prebiotic xylooligosaccharides on growth performances and digestive enzyme activities of allogynogenetic crucian carp (Carassius auratus gibelio), Fish Physiol. Biochem. 35 (2009) 351-357.

[77] S. Refstie, A.M. Bakke-McKellep, M.H. Penn, A. Sundby, K.D. Shearer $\AA$ A. Krogdahl, Capacity for digestive hydrolysis and amino acid absorption in Atlantic salmon (Salmo salar) fed diets with soybean meal or inulin with or without addition of antibiotics, Aquaculture 261 (2006) 392-406.

[78] J.-D. Ye, K. Wang, F.-D. Li, Y.-Z. Sun, Single or combined effect of fructo- and mannan oligosaccharide supplements and Bacillus clausii on the growth, feed utilization, body composition, digestive enzyme activity, innate immune response and lipid metabolism of the Japanese flounder (Paralichthys olivaceus), Aquac. Nutr. 17 (2011) e902-e911.

[79] M. Anguiano, C. Pohlenz, A. Buentello, D.M. Gatlin III, The effects of prebiotics on the digestive enzymes and gut histomorphology of red drum (Sciaenops ocellatus) and hybrid striped bass (Morone chrysops $\times$ M. saxatilis), Br. J. Nutr. 109 (2013) 623-629.

[80] A. Bonaldo, A.J. Roem, P. Fagioli, A. Pecchini, I. Cipollini, P.P. Gatta, Influence of dietary levels of soybean meal on the performance and gut histology of gilthead sea bream (Sparus aurata L.) and European sea bass (Dicentrarchus labrax L.), Aquac. Res. 39 (2008) 970-978. 